\title{
Petrografía de las pastas Cerámicas del sitio Las Garzas EN EL CONTEXTO DE LAS TRADICIONES ALFARERAS DEL PRIMER

\author{
milenio en el Noroeste Argentino
}

\author{
Vanesa Beatriz Juarez ${ }^{a}$ \\ María Beatriz Cremonte
}

\begin{abstract}
Resumen
Se muestran los resultados de la última investigación llevada a cabo sobre pastas de cerámicas provenientes de un sitio temprano (600 a.C.-650 d.C.) denominado Las Garzas, ubicado en el valle de Lerma (provincia de Salta, Argentina). Este abordaje implicó una revisión de los estudios previamente iniciados por Cremonte, lo cual permitió ajustar la petrografia de las pastas ampliando la muestra analizada e incorporando nuevos y diferentes procedimientos de registro. Los datos obtenidos en relación con la geología local permitieron plantear hipótesis sobre las procedencias de las vasijas estudiadas. Asimismo, la comparación con otros conjuntos identificados en el valle de Lerma, y en otras áreas más distantes como el valle de Tafí y San Francisco, posibilitó determinar nexos en las técnicas de manufactura que contribuyen al conocimiento de las tradiciones tecnológicas cerámicas tempranas en el Noroeste Argentino.
\end{abstract}

Palabras clave: Las Garzas, valle de Lerma, pastas cerámicas, petrografia, tecnologías

\section{Abstract \\ PETROGRAPHY OF THE CERAMIC PASTES FROM LAS GARZAS SITE IN THE CONTEXT OF THE POTTERY TRADITIONS OF THE FIRST MILLENNIUM IN NORTHWESTERN ARGENTINA}

We present the results about the last researches carried out on ceramic fabrics of Las Garzas, an Early site (600 a.C.650 d.C.) located in the Lerma Valley (Province of Salta, Argentina). This approach implied a revision of previous studies initiated by Cremonte (Cremonte et al., 1987), which allowed us to adjust the petrography of the ceramic pastes, expanding the analyzed sample and completing the previous analyzes incorporating different recording procedures. Data obtained in relation to the local geology allowed us to hypothesize about foreign or in situ production of the vessels represented in the studied sample. Likewise, comparisons with other pottery groups from the Lerma Valley and more distant pottery traditions such as Tafi and San Francisco valleys, allowed us to evaluate links in the manufacturing techniques that contribute to our knowledge about early pottery technological traditions in Northwestern Argentina.

Keywords: Las Garzas, Lerma Valley, ceramic pastes, petrography, technologies

a Instituto de Ecorregiones Andinas (INECOA-UNJu-CONICET) e Instituto de Datación y Arqueometría (InDyA-UNJu-UNT-CONICET-Gob. de Jujuy)

Correo electrónico: vanesa.juarez@conicet.gov.ar

${ }^{\mathrm{b}}$ Instituto de Ecorregiones Andinas (INECOA-UNJu-CONICET) e Instituto de Datación y Arqueometría (InDyA-UNJu-UNT-CONICET-Gob. de Jujuy)

Correo electrónico: cremontebeatriz@gmail.com 


\section{Introducción}

Los sitios del valle de Lerma presentan la particularidad de emplazarse en una zona del Noroeste Argentino (NOA) con características intermedias entre los dominios amazónico y andino. Esta particularidad ambiental constituye hoy —al igual que en otras áreas del NOA - una pieza clave para conocer y completar el entramado cultural prehispánico a nivel regional. Debido a diversos factores, tales como la escasa visibilidad de los asentamientos, ya sea por la existencia de monte, o por alteraciones producidas por explotaciones agrícolas y ganaderas modernas, lugares como estos fueron considerados como áreas "periféricas» en términos de un concepto equivalente a «secundarias o menos relevantes». Sin embargo, los avances en la arqueología del NOA han revalorizado estos ámbitos transicionales; por lo que necesitamos conocer sus idiosincrasias y tradiciones culturales en relación con sus paisajes, materialidades y eventos paleoambientales, para comprender la ocupación de sus territorios y los elementos constitutivos de su identidad.

Sobre la base de los estudios realizados en el sitio Las Garzas por Cremonte y colaboradores (Cremonte et al. 1987), decidimos encarar una revisión y ampliación del análisis cerámico en sintonía con los recientes avances logrados en la identificación de estilos y tradiciones alfareras del primer milenio de la Era, tanto en el NOA como en el área centro sur andina. Retomamos así el estudio de la alfarería enfatizando, en esta ocasión, el análisis de pastas en secciones delgadas, su relación con datos granulométricos y con formas específicas de vasijas. Esto permitió obtener un registro completo de estilos y/o tradiciones tecnológicas presentes en la alfarería del sitio, considerando las características de su producción local y la identificación de vasijas obtenidas mediante redes de interacción de alcance regional.

En el marco de los objetivos planteados, debemos aclarar que diferenciamos estilo de tradición, entendiendo al primero como un conjunto de elecciones técnicas realizadas durante la manufactura y uso de la cultura material. Dichas elecciones no son aleatorias, sino internalizadas durante el proceso de aprendizaje y compartidas tácitamente dentro de una comunidad (Lemonnier 1989; Dietler y Herbich 1998). El proceso que implica la transmisión de los conocimientos técnicos de generación en generación desemboca en la sedimentación histórica de los patrones que permite conformar tradiciones (Ingold 2000; Feely 2012; Pereyra Domingorena 2012). A diferencia de estas últimas, debe tenerse en cuenta que los estilos no son estáticos y pueden ocurrir cambios tecnológicos rápidos o graduales (Childs 1991).

\section{Las Garzas: un sitio Formativo en el valle de Lerma}

Las Garzas se ubica en el sector oriental del valle de Lerma, 52 kilómetros al Sur de la ciudad de Salta (Fig. 1). Fue localizado dentro de una finca que lleva el mismo nombre, a 500 metros del río Arias, gracias a las indicaciones brindadas por el Sr. Araya, un lugareño que residía en dicha finca realizando tareas agrícolas. El Sr. Araya poseía una acotada colección de materiales arqueológicos, especialmente cerámicos que aportaron información al inventario de formas derivado del análisis de los fragmentos recuperados en las excavaciones de campo (Fig. 2).

Los materiales registrados en las excavaciones realizadas en la década de los años 80 incluían fragmentos de cerámicas pintadas, con marleado y sin tratamientos de superficie; líticos como puntas de proyectil de obsidiana, lascas, núcleos, morteros y conanas; y restos faunísticos de peces, anfibios, valvas de moluscos de ambiente fluvial y mamíferos de pequeño y mediano tamaño, algunos de estos aparecieron termoalterados. Según las correspondencias que mostraron las tecnologías líticas y cerámicas provenientes del nivel superficial como subsuperficial, se trataría de un

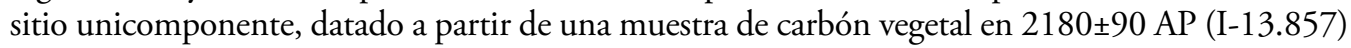
(Cremonte et al. 1987). 
Figura 1. Ubicación geográfica del sitio Las Garzas (extraido y modificado de Lazzari et al. 2009, Journal of Archaeological Science. Escala 1:50. Noroeste Argentino. Blanco y negro).

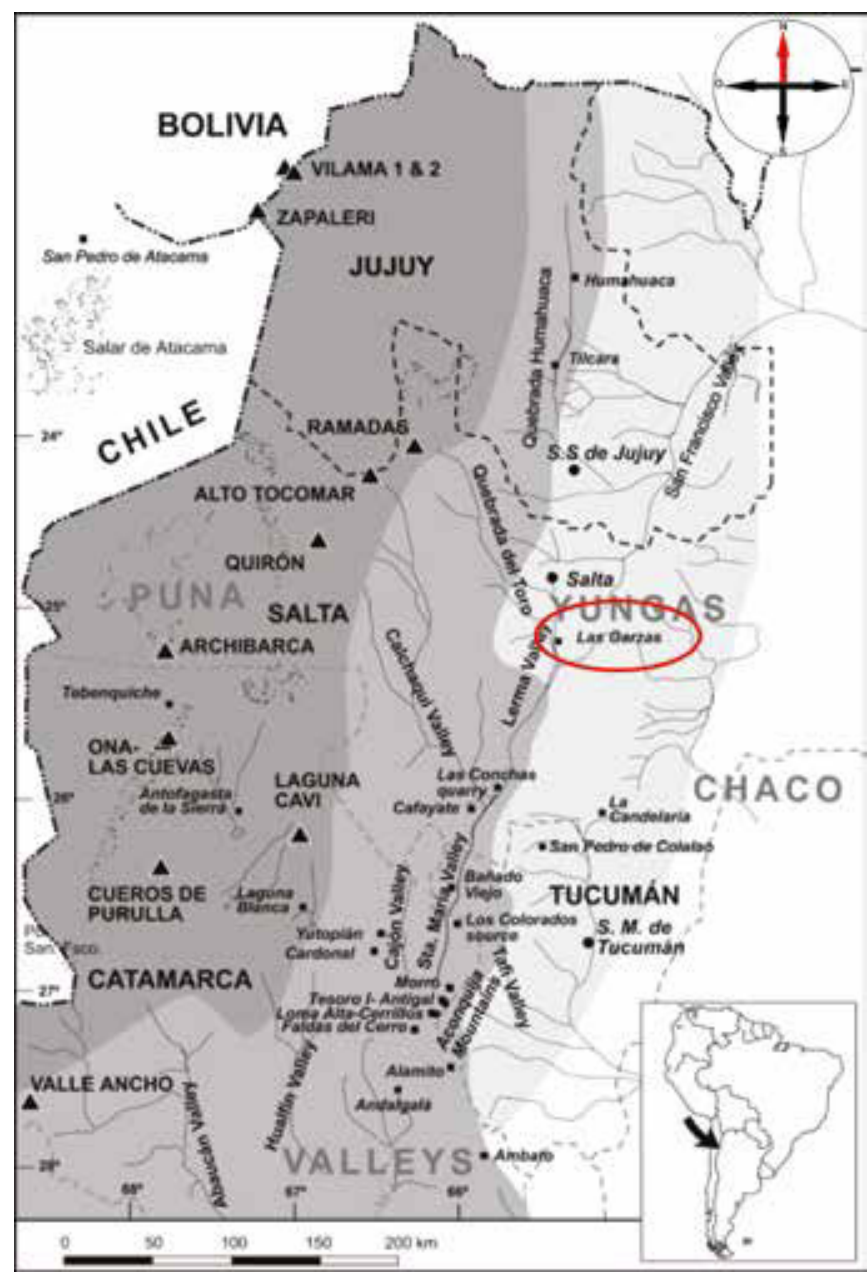

La naturaleza de los materiales arqueológicos recuperados refleja una amplia gama de actividades llevadas a cabo en el sitio relacionadas con un patrón de subsistencia diversificado. Se infiere que tales actividades implicaron la captura y recolección de recursos silvestres y también el probable cultivo de maíz o su obtención mediante intercambios (debido al uso de marlos como alisadores). Desde esta perspectiva, la caracterización del sitio nos permite hablar de un asentamiento ubicado en una zona de transición como es el valle de Lerma con áreas de baja elevación, serranías orientales, abras y quebradas, lo que habría favorecido el acceso a una amplia diversidad de recursos de diferentes ambientes.

En la Figura 2 se ilustran algunos ejemplares de la colección del señor Araya procedente de Las Garzas. A pesar de los años transcurridos, resulta muy difícil contextualizar a este sitio en el marco de la arqueología del valle de Lerma porque, lamentablemente, seguimos desconociendo los desarrollos culturales acaecidos en el mismo durante el primer milenio de nuestra era. Si bien, otros investigadores han explorado el área en la segunda mitad del siglo XX, no se ejecutaron investigaciones sistemáticas que permitieran obtener información más completa sobre las ocupaciones prehispánicas tempranas en el valle (González 1963; Escobar 1996; Baldini et al. 2003). Considerando el actual estado de la cuestión, apuntamos a colaborar en la integración de los datos hasta ahora disponibles y profundizar en los análisis de los materiales recuperados implementando nuevas estrategias, por ahora, aplicadas al estudio de la alfarería. 

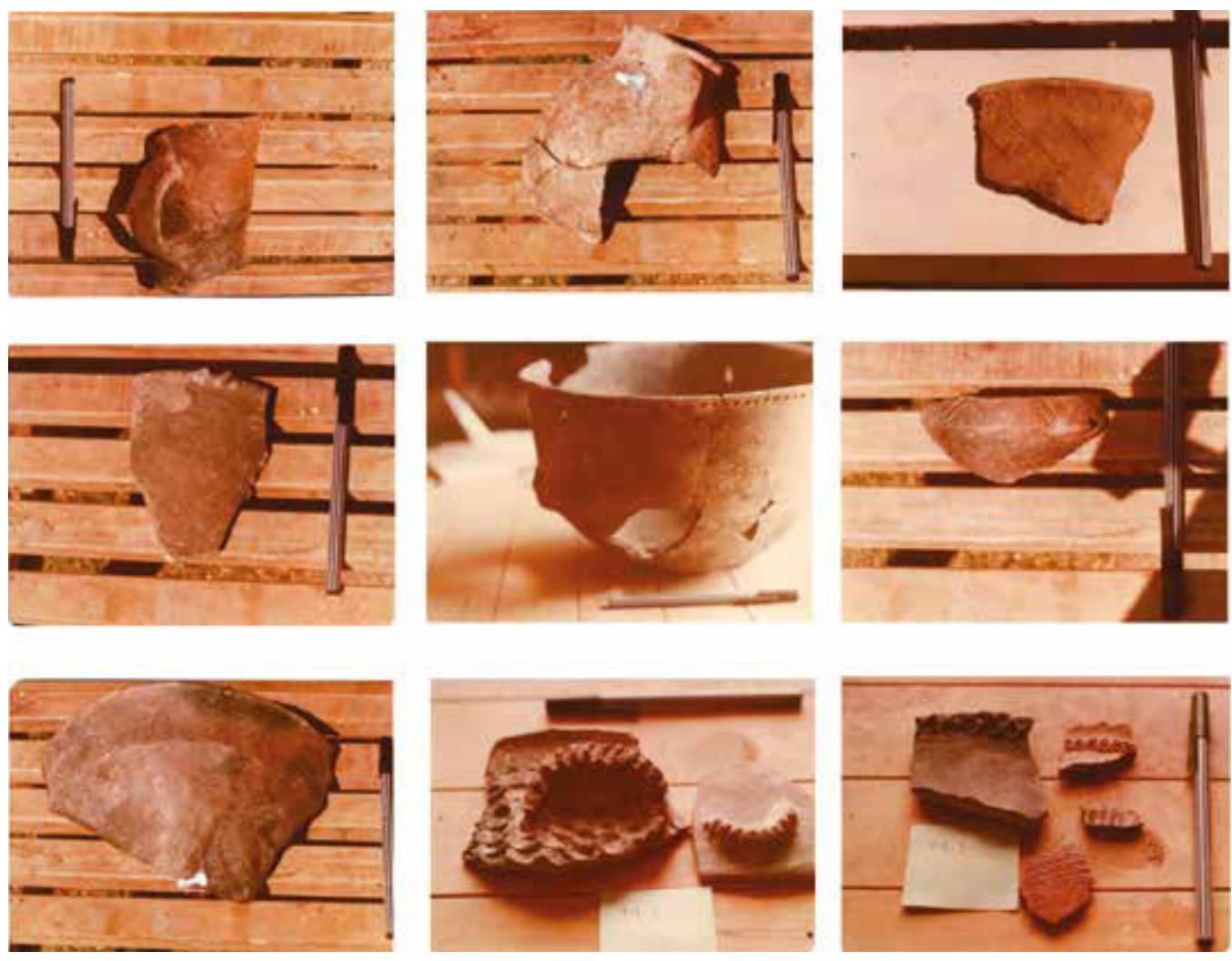

Figura 2. Colección Araya compuesta por un vaso cilíndrico, jarra, escudilla de contorno simple y angular, y también fragmentos con aplicación al pastillaje como decoración (Autores: Juarez y Cremonte).

\section{Entorno geológico del valle de Lerma}

El valle de Lerma se ubica dentro de la provincia geológica Cordillera Oriental (Turner 1972) y se caracteriza por la presencia de dos frentes fallados que delimitan el valle. Estos frentes son producto de la tectónica compresiva andina que empezaron a formarse después del Mioceno Tardío y ya existía para el Plio Pleistoceno. La columna estratigráfica del valle de Lerma y sus alrededores se resume de la siguiente manera: Grupo Lerma (Formación Sacha, Formación Las Tienditas, Formación Puncoviscana, Formación Corralito y Formación Gauchos); Grupo Mesón (Formación Lizoite, Formación Campanario, Formación Chalhualmayoc); Grupo Santa Victoria (Formacióon La Pedrera, Formación San José, Formación Caldera, Formación Floresta, Formación Áspero, Formación San Bernardo, Formación Mojotoro, Formación Santa Gertrudis); Grupo Salta; Subgrupo Pirgua (Formación La Yesera, Formación Las Curtiembres, Formación Los Blanquitos); Subgrupo Balbuena (Formación Lecho, Formación Yacoraite, Formación Tunal); Subgrupo Santa Bárbara (Formación Mealla, Formación Maíz Gordo, Formación Lumbrera); Grupo Orán; Subgrupo Metán (Formación Río Seco, Formación Anta, Formación Jesús María); Subgrupo Jujuy (Formación Guanuco, Formación Piquete); Cuaternario (Formación Calvimonte, Formación Tajamar, Formación La Viña) (Turner op cit.).

La geología descrita corresponde a todo el valle de Lerma, el cual presenta una extensión longitudinal de 100 kilómetros aproximadamente y 30 kilómetros en el sector más ancho. Sin embargo, en los que respecta a las inmediaciones del sitio Las Garzas, la geología se caracteriza por presentar a los grupos Mesón y Salta, como así también depósitos Cuaternarios, en los cuales es factible 
encontrar: wackes; gravas medianas y gruesas, areniscas, cuarzos, cuarcitas medianas y finas (rosadas y blanquecinas); arenas finas y medianas, limolitas cuarzosas y micáceas (verdes y moradas); limolitas arcillosas (pardo-rojizo oscuro laminadas), pelitas (castaño claro, rosado, naranja grisáceo ocres, amarillentas y verdosas), calizas, arcillas y basalto Las Conchas (Turner 1972; Larcher 2006).

\section{El universo cerámico: metodología de análisis}

Al no registrarse piezas completas durante la prospección y excavación del sitio, trabajamos con los fragmentos cerámicos hallados, los cuales fueron agrupados en familias de fragmentos teniendo en cuenta similitudes en características como color, tratamiento de superficie, espesor y cocción. Con el propósito de reconstruir la diversidad morfológica de las piezas remontadas realizamos análisis cualitativos y cuantitativos, recurriendo al reconocimiento de grupos morfológicos generales propuestos por Balfet et al. (1983) y Cremonte y Bugliani (2006-9). Así, cada grupo fue posteriormente subdividido en variedades de acuerdo a puntos singulares presentes en el perfil y a características del borde y cuello de las vasijas.

Para el análisis petrográfico seleccionamos una muestra de 18 secciones delgadas sobre la base de una clasificación previa de pastas en lupa binocular $(10$ a 40x) y de su covariación con estados de atributos de tratamiento de las superficies, espesor de las paredes e indicadores morfométricos (Tabla 1). A las 18 secciones delgadas agregamos cinco procedentes de otros sitios tempranos del NOA, con las cuales pudimos establecer similitudes teniendo en cuenta las caracterizaciones petrográficas, la distribución modal (point counting) y el análisis multivariado mediante la utilización del programa PAST 2.17 (Bugliani 2009). La caracterización petrográfica se realizó en un microscopio de polarización Leica DM750 P.

Como puede observarse en la Tabla 1, en la selección de los fragmentos para secciones delgadas se puso énfasis en el estilo Vaquerías. La razón de ello se basa en que la abundancia relativa de este estilo en el sitio es llamativa, una característica poco frecuente en el NOA donde la densidad de fragmentos Vaquerías es siempre muy baja. En razón de ello, Las Garzas ofrece una situación especial para indagar aspectos tecnológicos de su producción como así también para evaluar un posible caso de locus de producción local de Vaquerías, ya que en su gran mayoría son siempre de manufactura no local.

El análisis petrográfico se basó en la caracterización cualitativa y cuantitativa de los componentes de las pastas cerámicas. Las fases plásticas, como los elementos no plásticos menores a 15 micras, fueron consideradas como parte de la matriz arcillosa teniéndose en cuenta coloración y estructura. Posteriormente se determinó la mineralogía de las inclusiones mayores a 15 micrones distinguiendo entre cristaloclastos y litoclastos. Se identificaron minerales opacos, vidrio volcánico, nódulos arcillosos y tiesto molido. Los tamaños de las inclusiones no plásticas fueron estimados midiendo el eje longitudinal máximo de la partícula o grano con la ayuda de un ocular micrométrico y posteriormente fueron clasificados según la escala granulométrica de Wentworth (tomada de Adams et al. 1984), teniendo en cuenta tres rangos referenciales: arena fina $(62-249 \mu \mathrm{m})$; arena media $(250-499 \mu \mathrm{m})$; arena gruesa y muy gruesa $(500-2000 \mu \mathrm{m})$. Dichos agrupamientos se realizaron considerando los promedios de las inclusiones no plásticas representativas de cada pasta que se presentaban en porcentajes superiores al 5\% (Cremonte y Bugliani 2006-2009). Finalmente, se efectuó un conteo mínimo de 300 puntos por sección delgada, lo que permitió, junto con los anteriores procedimientos, caracterizar la pasta cerámica en cuanto a estructura de fondo de la matriz arcillosa, la identificación, cuantificación y determinación de tamaños de materiales no plásticos y cavidades. 
Tabla 1. Descripción de los 18 fragmentos cerámicos seleccionados para el análisis petrográfico (Autores: Juarez y Cremonte).

\begin{tabular}{|c|c|c|}
\hline Sitio & & Las Garzas \\
\hline Corte & Procedencia & Características tipológicas \\
\hline 1 & $\mathrm{LG} / 5-5.10$ & Vaso negro amarronado pulido \\
\hline 2 & LG/TAT 139.18 & Botellón calceiforme negro amarronado pulido \\
\hline 3 & LG/O/IV.2.Potrero Sur & Fragmento pintado Negro/Rojo. \\
\hline 4 & LG54-A5-NO-C3- 167 & Fragmento pintado rojo pulido \\
\hline 5 & LG Superficie & Vasija sub-globular ordinaria micácea \\
\hline 6 & LG Superficie & Base de cuenco Vaquerías. Pintado Negro/Beige \\
\hline 7 & LG Superficie & Fragmento con improntas en superficie externa \\
\hline 8 & LG Superficie & Vasija sub-globular gris negro alisado \\
\hline 9 & LG Superficie & Olla negra pulida con apéndice troncocónico \\
\hline 10 & LG Superficie & Vasija sub-globular castaño claro alisado \\
\hline 11 & LG/ B5 Nº 46 & Fragmentos Vaquerías \\
\hline 12 & LG O/III N ${ }^{\circ} 1$ & Fragmentos Vaquerías \\
\hline 13 & LG O/III N ${ }^{\circ} 9$ & Fragmentos Vaquerías. Blanco/Rojo oscuro \\
\hline 14 & LG O/III N² 253 & Fragmentos Vaquerías. Rojo /Beige \\
\hline 15 & LG O/III No25 & Fragmentos Vaquerías Polícromo. \\
\hline 16 & LG O/IV N²09 & Fragmentos Vaquerías. Rojo/ Beige. \\
\hline 17 & LG O/II N63 & Fragmentos Vaquerías. Beige. \\
\hline 18 & LG O/IV N 183 & Fragmentos Vaquerías Polícromo. \\
\hline
\end{tabular}

\section{Resultados}

El corpus cerámico de Las Garzas recuperado en recolecciones de superficie y áreas excavadas es restringido, recuperándose 305 fragmentos, a partir de los cuales identificamos 88 familias de fragmentos, algunas remontables, llegando a conocer 9 formas: piezas abiertas (vaso cilíndrico con asa lateral, escudilla semiesférica de contorno simple; escudilla de perfil compuesto con punto angular en el tercio inferior de la altura de la pieza y paredes levemente cóncavas) y piezas cerradas (botellón calceiforme; vasija subglobular; olla con apéndice troncocónico; olla) (Fig. 3).

Durante el análisis microscópico de los cortes delgados $(\mathrm{CD})$ de las pastas logramos diferenciar antiplásticos, cavidades $(\mathrm{C})$ y matriz $(\mathrm{Mz})$. Como antiplástico identificamos litoclastos y cristaloclastos de: cuarzo $(\mathrm{Qz})$, plagioclasa $(\mathrm{Pg})$, biotita $(\mathrm{Bi})$, muscovita $(\mathrm{Mu})$, opacos $(\mathrm{Op})$, pizarra $(\mathrm{Pz})$, limolita $(\mathrm{Lm})$, vulcanita $(\mathrm{Vc})$ y granito (Gto). Las inclusiones cuarzo, pizarra, limolita y tiesto molido $(\mathrm{T})$ se registraron en gran parte de las pastas analizadas en porcentajes superiores al 5\%, mientras que el granito apareció en igual proporción, aunque excepcionalmente en dos secciones (5 y 20) (Tabla 2). 

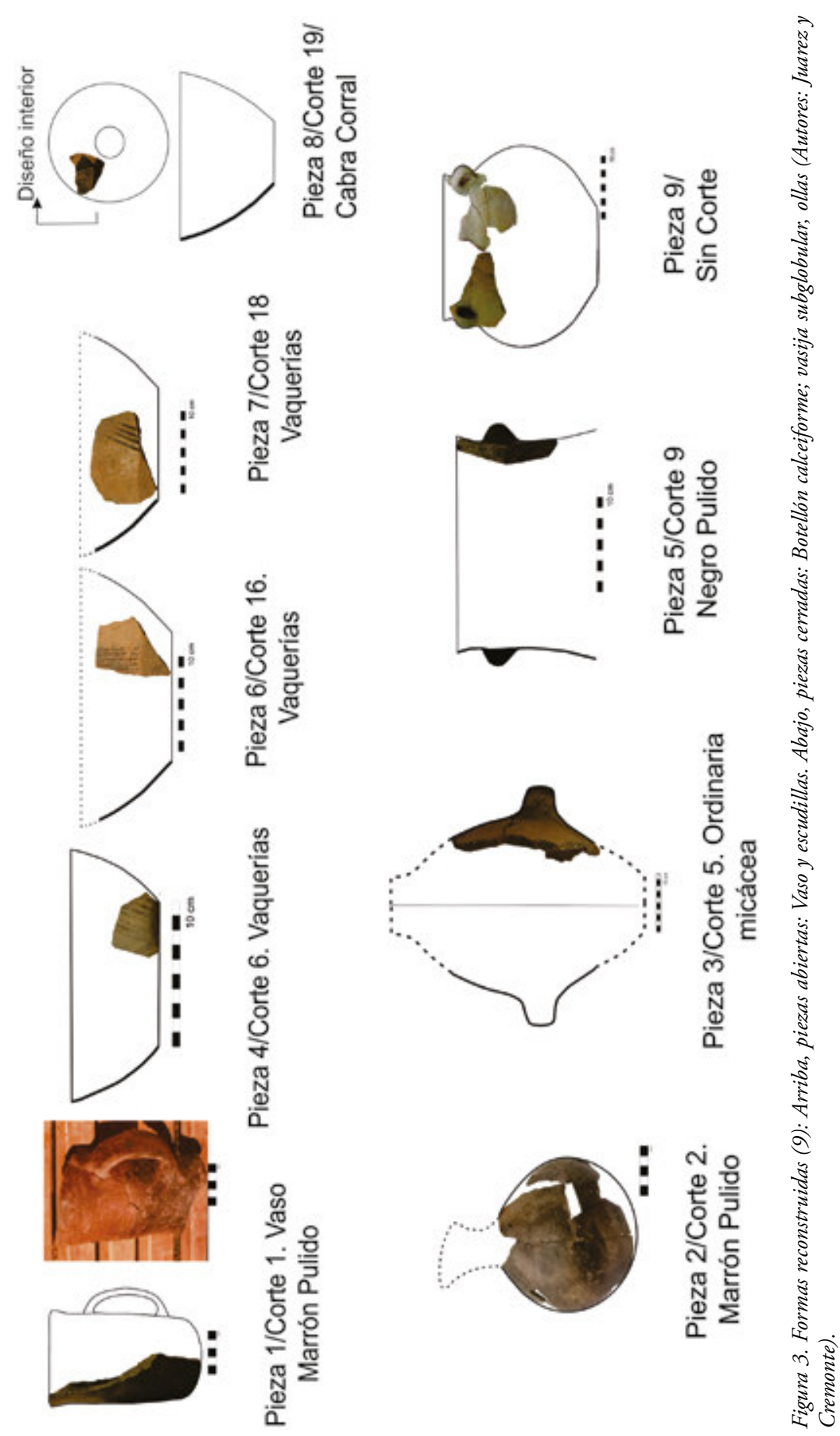
Tabla 2. Componentes de las pastas cerámicas. Distribución modal mediante point counting (Autores: Juarez y Cremonte).

\begin{tabular}{|c|c|c|c|c|c|c|c|c|c|c|c|c|c|c|c|c|c|c|c|}
\hline CD & $\mathrm{Mz}$ & C & $\mathrm{Qz}$ & $\mathrm{Pg}$ & $\mathrm{Bi}$ & $\mathrm{Mu}$ & Op & Ox & $\mathrm{Pz}$ & $\mathrm{Lm}$ & Are & $Q t$ & $\mathrm{Vc}$ & $\mathrm{CO}_{3} \mathrm{Ca}$ & Gt & $\begin{array}{l}\mathrm{N} . \\
\text { Arc }\end{array}$ & $\mathrm{T}$ & $\begin{array}{c}\mathrm{An} / \\
\mathrm{Px}\end{array}$ & $\%$ \\
\hline 1 & 64 & 6.5 & 14 & 0.3 & 0.9 & 1.8 & 2.4 & 1.5 & 4.1 & 2.7 & 0.9 & 0 & 0 & 0 & 0 & 1.47 & 0 & 0.29 & 100 \\
\hline 2 & 56 & 5.6 & 5 & 0.3 & 0.3 & 0 & 2.2 & 0.6 & 26 & 3.1 & 0 & 0 & 0 & 0 & 0 & 0.93 & 0 & 0.31 & 100 \\
\hline 3 & 68 & 7.3 & 1.7 & 0.7 & 0 & 0.4 & 1.1 & 2.8 & 15 & 1.7 & 0 & 0 & 0 & 0 & 0 & 1.74 & 0 & 0 & 100 \\
\hline 4 & 53 & 11 & 6.1 & 0.6 & 0.3 & 1 & 0.6 & 0.6 & 11 & 4.4 & 0 & 1 & 2.6 & 0.32 & 0 & 0.64 & 7.1 & 0 & 100 \\
\hline 5 & 55 & 7.8 & 12 & 3 & 4.1 & 2.2 & 0.5 & 0.3 & 0 & 0 & 0 & 0 & 0 & 0 & 14 & 0.54 & 0 & 0.27 & 100 \\
\hline 6 & 69 & 3.2 & 8.7 & 1.5 & 0.3 & 0.6 & 1.5 & 1.5 & 0.9 & 0.9 & 0 & 0.3 & 0 & 0 & 0 & 0.9 & 10 & 0 & 100 \\
\hline 7 & 64 & 8.7 & 5.2 & 0.6 & 0 & 0 & 2.6 & 2.6 & 7.2 & 2 & 0 & 0 & 0 & 0 & 0 & 1.73 & 5.8 & 0 & 100 \\
\hline 8 & 39 & 7.9 & 5.6 & 0 & 0 & 0 & 1.6 & 0.7 & 29 & 10 & 5.6 & 0.7 & 0 & 0 & 0 & & 0 & 0 & 100 \\
\hline 9 & 60 & 5.4 & 11 & 1.2 & 3 & 0 & 1.8 & 0.9 & 4.8 & 3.9 & 0 & 0.6 & 0 & 0 & 0 & 1.2 & 6.3 & 0 & 100 \\
\hline 10 & 55 & 6.2 & 2.4 & 0 & 0.3 & 0.3 & 0.8 & 0.5 & 20 & 10 & 0 & 0.8 & 0 & 1.08 & 0 & 0 & 0 & 0 & 100 \\
\hline 11 & 62 & 13 & 6 & 1.4 & 1.1 & 0 & 2.1 & 2.1 & 7.4 & 1.8 & 0 & 0 & 0 & 0 & 0 & 2.83 & 0 & 0 & 100 \\
\hline 12 & 58 & 10 & 5.2 & 1.5 & 3.5 & 0 & 0.9 & 0.9 & 1.4 & 0 & 4 & 0 & 0 & 0 & 0 & 0.75 & 13 & 0 & 100 \\
\hline 13 & 52 & 9 & 3.5 & 0 & 3.1 & 0 & 1.4 & 1.4 & 0.7 & 7.6 & 3.5 & 0 & 0 & 0 & 0 & 0 & 18 & 0 & 100 \\
\hline 14 & 71 & 9.2 & 1.2 & 0.3 & 0 & 0 & 0.3 & 0 & 5.9 & 1.8 & 1.2 & 0.3 & 0 & 0 & 0 & 0.87 & 7.7 & 0 & 100 \\
\hline 15 & 73 & 10 & 3.2 & 0 & 0 & 0 & 0 & 0.3 & 4.4 & 3.8 & 0 & 0 & 0 & 0 & 0 & 1.58 & 3.8 & 0 & 100 \\
\hline 16 & 65 & 11 & 8.6 & 1.3 & 2.4 & 0 & 2.1 & 1.1 & 3.2 & 3.2 & 0 & 0 & 0 & 0 & 0 & 2.68 & 0 & 0 & 100 \\
\hline 17 & 62 & 11 & 4 & 0.8 & 1.3 & 0 & 1.6 & 1.6 & 6.4 & 4.2 & 0.5 & 0.5 & 0 & 0 & 0 & 2.64 & 3.7 & 0 & 100 \\
\hline 18 & 76 & 5.2 & 2.2 & 0.5 & 0.5 & 0 & 1 & 1 & 1 & 0.5 & 0 & 0 & 0 & 0 & 0 & 2.2 & 9.8 & 0 & 100 \\
\hline $\begin{array}{c}\text { CC. } \\
\text { Lav. } 1 \\
\end{array}$ & 52 & 13 & 3.6 & 0.5 & 2.8 & 0 & 1.4 & 2.1 & 7.3 & 6.6 & 1.2 & 0 & 0 & 0 & 0 & 0 & 9.5 & 0 & 100 \\
\hline
\end{tabular}

Mediante el tratamiento estadístico multivariado (Análisis de Conglomerados) de los datos cuantitativos de la Tabla $2(\mathrm{~N}=23)$ aplicando el programa Past 2.17 y del método Ward obtuvimos el fenograma (Fig. 4). En el mismo se discriminaron seis (6) agrupamientos y dos (2) variedades que reflejan las similitudes y disimilitudes petrográficas y texturales de las pastas que conforman la muestra, coincidiendo con lo observado en microscopio (Fig. 5).

Los grupos de pastas identificados presentan las siguientes características:

LG1 (Tabla 3): Pertenecen a este grupo los cortes: 2. Las Garzas negro amarronado alisado; 10. Las Garzas castaño claro alisado; y 8. Las Garzas gris negro alisado.

Tabla 3. Pastas con abundante pizarra y cantidad variable de limolita cuarzosa (Autores: Juarez y Cremonte).

\begin{tabular}{|c|c|c|c|c|c|}
\hline Matriz & Cavidades & Pizarra & Lim. Cuarzosa & Cuarzo & Otros \\
\hline $40-56 \%$ & $6-8 \%$ & $20-28,50 \%$ & $3-13 \%$ & $2-5 \%$ & Ausentes o muy escasos \\
\hline
\end{tabular}


LG2 (Tabla 4): A este grupo corresponde los cortes: 5. Las Garzas rosado alisado y 20. La Ciénaga IRRC (del Norte del valle de Tafí).

Tabla 4. Pastas con litoclastos graniticos, abundantes cavidades y cuarzo (Autores: Juarez y Cremonte).

\begin{tabular}{|c|c|c|c|c|c|c|}
\hline Matriz & Cavidades & Granito & Cuarzo & Plagioclasa & Biotita & Muscovita \\
\hline $47-55 \%$ & $8-17 \%$ & $8-14 \%$ & $12-17 \%$ & $3 \%$ & $2-4 \%$ & $2-3.5 \%$ \\
\hline
\end{tabular}

LG3 (Tabla 5): Pertenecen a este grupo los cortes: 4. Las Garzas rojo pulido; 19. Cabra Corral Lav.1 (Valle de Lerma). Vaquerías. negro y rojo/ fondo sin pintar; 12. Las Garzas Vaquerías; y 13. Las Garzas Vaquerías blanco/rojo.

A pesar de las similitudes observadas, el corte 4, Rojo Pulido, presenta 2,54\% de litoclastos de vulcanitas (riodacita e ignimbrita), por ello consideramos que debe ser clasificado como una variedad dentro del grupo: LG3.Var 1.

Tabla 5. Pastas con tiesto molido agregado, cantidades variables de pizarra y limolita (Autores: Juarez y Cremonte).

\begin{tabular}{|c|c|c|c|c|c|}
\hline Matriz & Cavidades & Tiesto M. & Cuarzo & Pizarra & Limolita \\
\hline $52-58 \%$ & $6-12 \%$ & $7-18 \%$ & $3-6 \%$ & $1.5-11 \%$ & $0-7 \%$ \\
\hline
\end{tabular}

LG4 (Tabla 6): A este grupo pertenecen los cortes: 18. Las Garzas Vaquerías; 22. San Francisco bicolor; 23. San Francisco bicolor; y 6. Las Garzas. Vaquerías negro/ beige.

Tabla 6. Pastas finas con abundante tiesto molido incorporado (Autores: Juarez y Cremonte)

\begin{tabular}{|c|c|c|c|}
\hline Matriz & Cavidades & Tiesto $\mathrm{M}$. & Otros \\
\hline $70-80 \%$ & $3-7 \%$ & $10-13 \%$ & Ausentes o muy escasos. \\
\hline
\end{tabular}

LG5 (Tabla 7): Pertenecen a este grupo los cortes: 9. Las Garzas gris negro pulido; 1. Las Garzas marrón negro pulido; 16. Las Garzas Vaquerías; 11. Las Garzas Vaquerías; 7. Las Garzas ordinario; y 17. Las Garzas Vaquerías.

Las muestras 9, 1 y 16 conforman un subgrupo por presentar los porcentajes más elevados de cuarzo. Los porcentajes variables, en general muy bajos; de tiesto molido no permiten plantear el agregado intencional de los mismo, es decir que su presencia sería accidental.

Tabla 7. Pastas con cantidades variables de cuarzo y pizarra, y limolita cuarzosa en menor proporción (Autores: Juarez y Cremonte).

\begin{tabular}{|c|c|c|c|c|c|}
\hline Matriz & Cavidades & Cuarzo & Pizarra & Limolita cuarzosa & Tiesto M. \\
\hline $59-65 \%$ & $5-11 \%$ & $4-13 \%$ & $3-7 \%$ & $2-4 \%$ & $0-6 \%$ \\
\hline
\end{tabular}

LG6 (Tabla 8): Pertenecen a este grupo los cortes: 21. La Ciénega (norte valle de Tafi). Vaquerías; 14. Las Garzas Vaquerías; y 15. Las Garzas Vaquerías.

La pasta 3. Las Garzas pintado rojo alisado presenta $15 \%$ de litoclastos de pizarra, por ello consideramos que debe ser clasificada como una variedad dentro del grupo: LG6.Var 1.

Tabla 8. Pastas finas con mayor presencia de pizarra por sobre los otros componentes (Autores: Juarez y Cremonte).

\begin{tabular}{|c|c|c|c|c|c|c|c|c|}
\hline Matriz & Cavidades & Pizarra & Cuarzo & Plagioclasa & Biotita & Muscov & Lim. cuarzosa & Tiesto M. \\
\hline $71-73 \%$ & $14 \%$ & $5-6 \%$ & $1-3 \%$ & $<1 \%$ & $<1 \%$ & $<1 \%$ & $0-4 \%$ & $0-7 \%$ \\
\hline
\end{tabular}




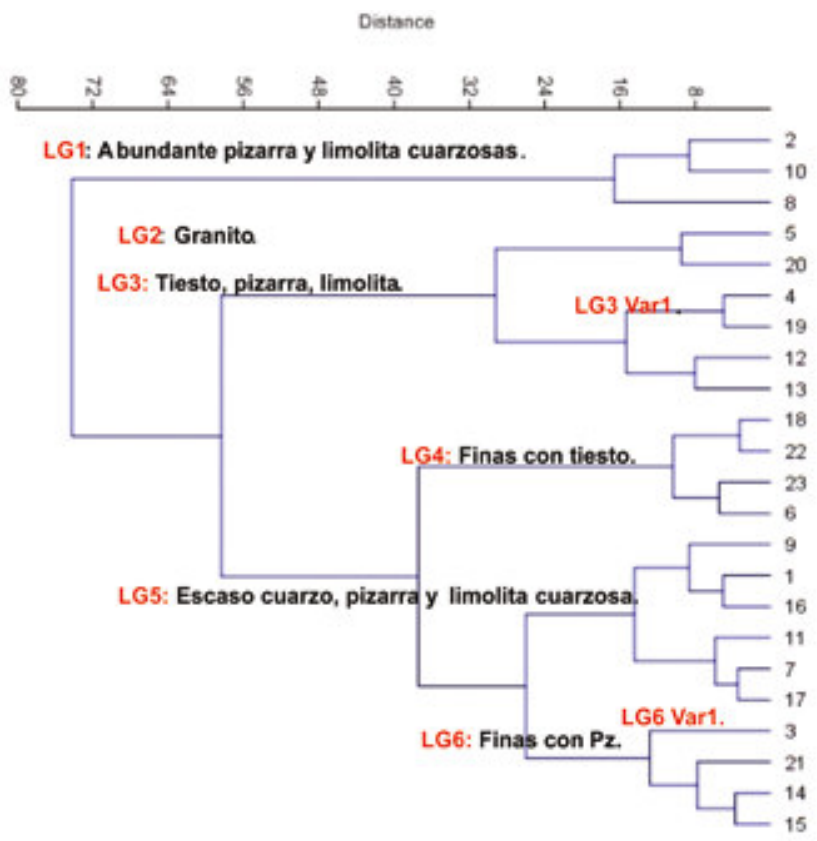

Figura 4. Grupos y variedades de las pastas cerámicas del sitio Las Garzas. Cluster Analysis. Programa PAST, versión 2.17 (Autores: Juarez y Cremonte).

Respecto del análisis granulométrico que formó parte del estudio petrográfico de las pastas, siguiendo los rangos referenciales arena fina; arena media; arena gruesa y muy gruesa, fue posible identificar tres agrupamientos (Tabla 9) (Fig. 6).

Tabla 9. Agrupamientos granulométricos identificados. Tamaño Arena Fina: 62-249 $\mu m$; Tamaño Arena Media: 250-499 um; Tamaño Arena Gruesa y Muy Gruesa: 500- 2000 uM (Autores: Juarez y Cremonte).

\begin{tabular}{ccc}
\hline Grupo Fino & Grupo Medio & Grupo Grueso \\
\hline (Tamańo Arena Fina) & (Tamaño Arena Media) & (Tamaño Arena Gruesa) \\
\hline LG1 & LG4 & LG2 \\
LG6 & LG17 & LG3 \\
LG9 & & LG5 \\
LG12 & LG7 \\
LG15 & LG8 \\
LG16 & LG10 \\
LG18 & & LG11 \\
& & LG13 \\
& & LG14
\end{tabular}




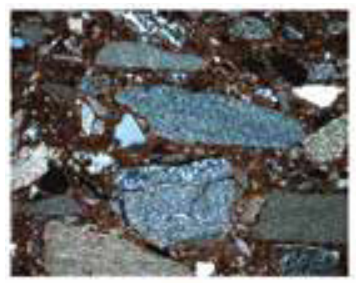

LG 1
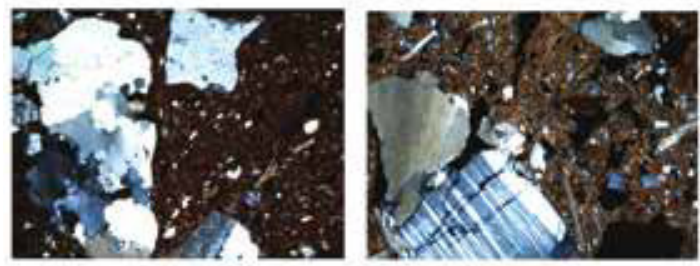

LG 2
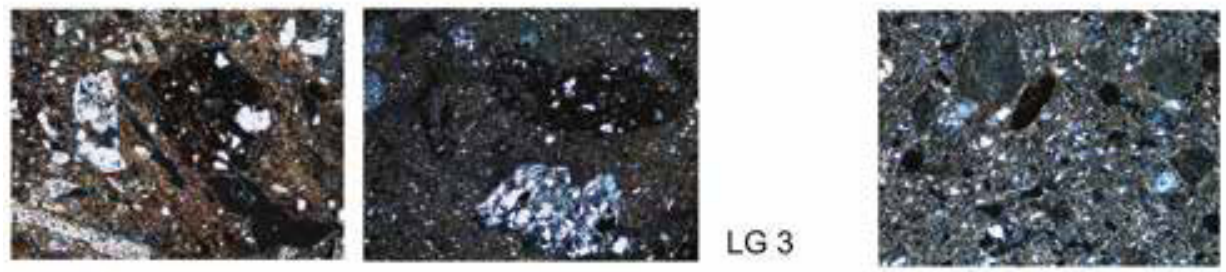

LG 3-Var 1
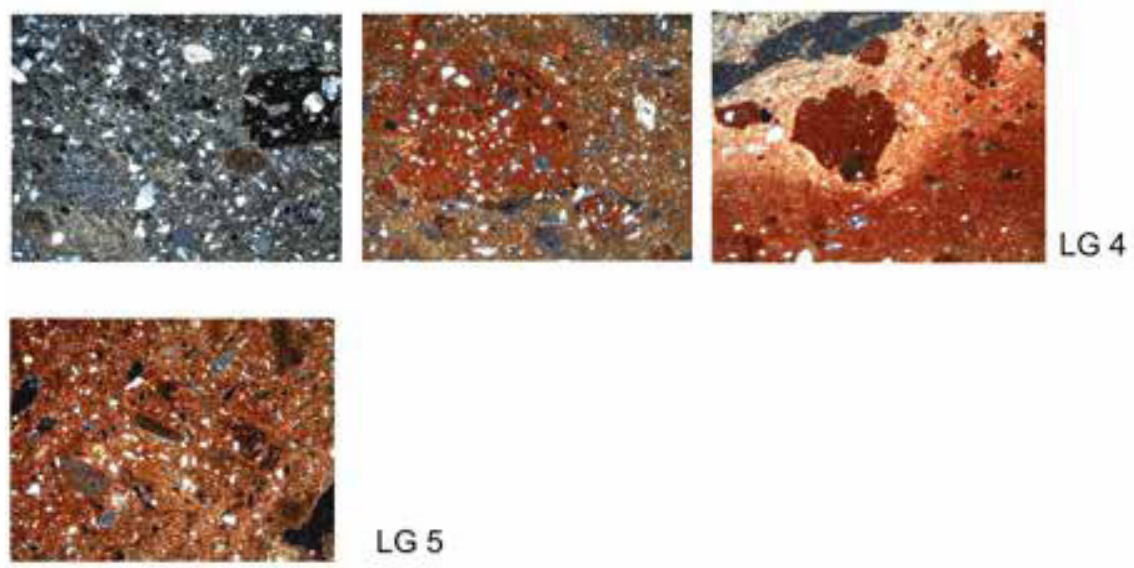

LG 5
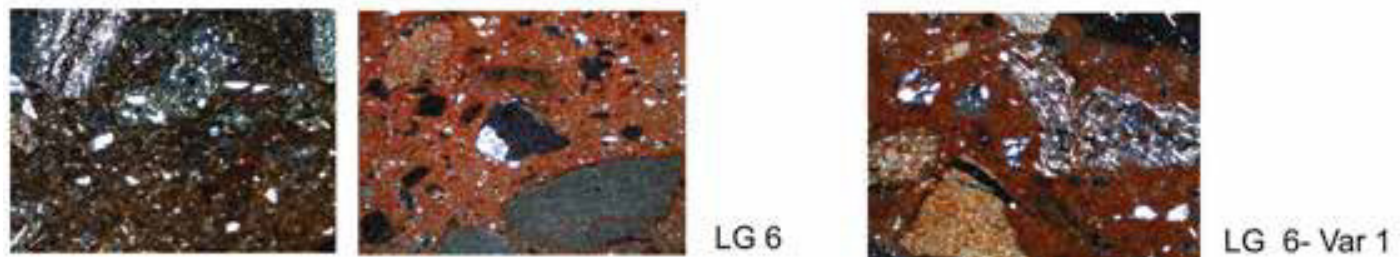

Figura 5. Fotomicrografias en nicoles cruzados (magnif.60x) de las pastas cerámicas representativas de cada grupo y variedad del sitio Las Garzas (Autores: Juarez y Cremonte). 
Respecto del análisis granulométrico que formó parte del estudio petrográfico de las pastas, siguiendo los rangos referenciales arena fina; arena media; arena gruesa y muy gruesa, fue posible identificar tres agrupamientos (Tabla 9) (Fig. 6).

Tabla 9. Agrupamientos granulométricos identificados. Tamaño Arena Fina: 62-249 $\mu m$; Tamaño Arena Media:

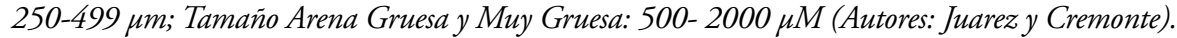

\begin{tabular}{ccc}
\hline Grupo Fino & Grupo Medio & Grupo Grueso \\
\hline (Tamańo Arena Fina) & (Tamańo Arena Media) & (Tamańo Arena Gruesa) \\
\hline LG1 & LG4 & LG2 \\
LG6 & LG17 & LG3 \\
LG9 & & LG5 \\
LG12 & LG7 \\
LG15 & LG8 \\
LG16 & LG10 \\
LG18 & LG11 \\
& & LG13 \\
& & LG14 \\
\hline
\end{tabular}

Como se puede ver en la Figura 7, el análisis granulométrico nos permitió corroborar el agregado de litoclastos y cristaloclasos como material antiplástico, en el 95.65\% de las pastas, evidenciado en la existencia de una distribución bimodal en los tamaños de las inclusiones no plásticas. El $4.35 \%$, representado en el corte 15 , también presenta una distribución bimodal y podría considerarse, según esta particularidad, como un agregado intencional a la pasta, sin embargo, los cristaloclastos y litoclastos identificados se registran en abundancia inferior al $5 \%$ por lo que descartamos esa posibilidad y contemplamos una incorporación accidental de los mismos.

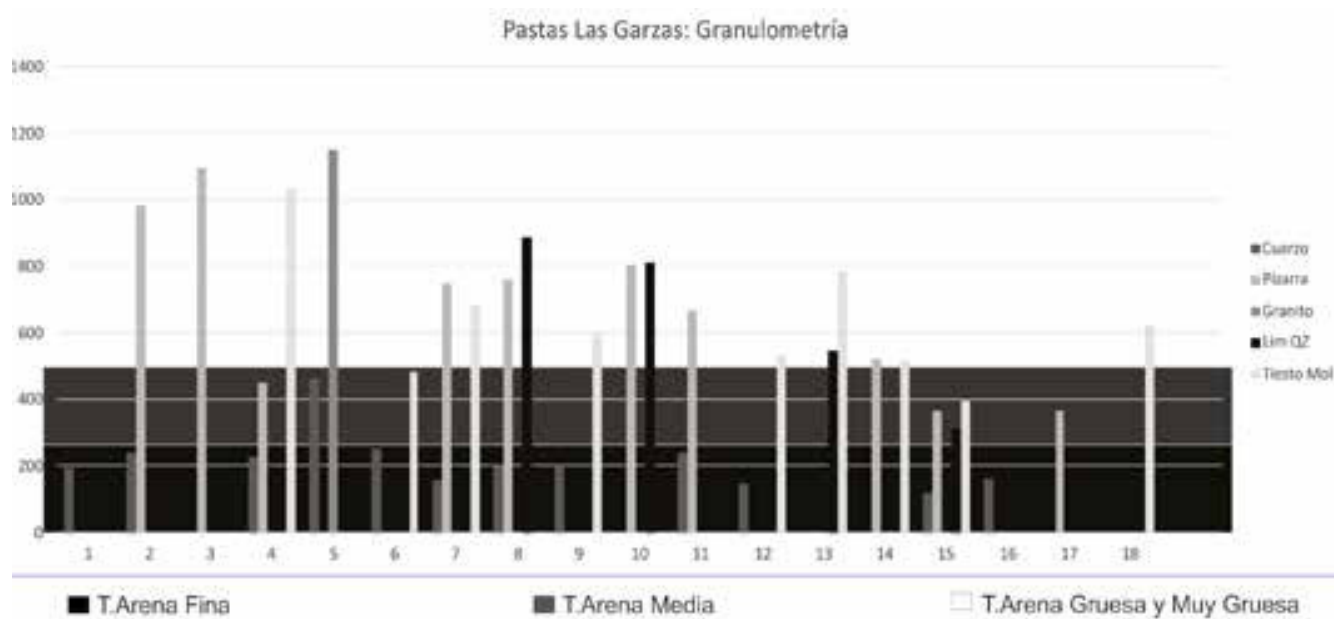

Figura 6. Representatividad de los tres grupos granulométricos diferenciados (Autores: Juarez y Cremonte).

Por otro lado, las variaciones registradas en los rangos de tamaños, fueron correlacionadas con las variables de formas y tamaños de las vasijas. Como resultado pudimos ver, en los casos en que las 
pastas presentaban agregado de pizarra, limolita y tiesto molido en fracción gruesa, que se correlacionaban directamente con la manufactura de piezas de gran porte y paredes gruesas. Por su parte, las fracciones gruesas de tiesto molido suelen presentarse frecuentemente en las escudillas de estilo Vaquerías recuperadas en Las Garzas.

Por último, debemos destacar que en Las Garzas no está presente la serie gruesa y la serie fina de pastas correspondientes respectivamente a vasijas de preparación y almacenaje por un lado y de servicio por el otro como sucede en los sitios tempranos de Tafí, Falda del Aconquija, Valle del Cajón (Scattolin y Gero 1999; Pereyra Domingorena 2012).

\section{Discusión y conclusiones}

Los resultados obtenidos ofrecen un panorama más completo y detallado del corpus cerámico de Las Garzas, siendo ahora comparable con los contextos alfareros de otros asentamientos contemporáneos del NOA. Obtuvimos caracterizaciones más precisas de las pastas y morfometría de las vasijas e indagamos aspectos de la producción alfarera en un componente cultural prácticamente desconocido en la región, a diferencia de lo que se conoce para sitios aledańos como Las Cuevas, San Francisco, Pampa Grande, Tafí y Candelaria (Ambrosetti 1906; Ryden 1934; González y Núñez Regueiro 1960; Heredia 1970; Dougherty 1975).

Las pastas de Las Garzas han sido comparadas con secciones delgadas, confeccionadas por Pantorrilla Rivas en 2015, correspondientes a fragmentos de sitios del valle de Lerma, específicamente del área perilago de Cabra Corral. También fueron comparadas con secciones delgadas de cerámicas de la tradición Tafí pertenecientes a sitios de la quebrada La Ciénega y del mismo valle de Tafí (Cremonte 1997), en la provincia de Tucumán, y con cerámicas de la tradición San Francisco procedentes de sitios del valle homónimo estudiados por la Dra. Gabriela Ortiz (Ortíz 2013), así como de las ocupaciones registradas en el sector centro sur de la Quebrada de Humahuaca (Garay de Fumagalli y Cremonte 2002).

Como resultado de la comparación, observamos un bajo grado de afinidad petrográfica indicando que la cerámica de Las Garzas presenta mayoritariamente idiosincrasias locales de manufactura, dictadas por las características de las materias primas empleadas. Las pocas conexiones registradas se vinculan con las pastas que habíamos advertido como de manufactura no local. Esta falta de uniformidad tecnológica confirma algo que venimos viendo en los contextos alfareros del Formativo en el NOA: las improntas fuertemente locales en los comportamientos de manufactura. Esto no se contradice con el amplio y creativo espectro de atributos morfológicos y decorativos que, en general ostentan las alfarerías tempranas del NOA concebidas en el seno de poblados dispersos con baja densidad de habitantes, en la mayoría de los casos autosuficientes y ligados a una economía casi exclusivamente de subsistencia.

Según las potencialidades económicas y diversidad de materias primas disponibles en Las Garzas, así como en su ubicación en un locus de mayor o menor tráfico intra e interregional, podemos intentar explicar las expresiones tecnológicas y artísticas de su alfarería. Así, por ejemplo, alfarerías muy tempranas como las de Cueva de Cristóbal (Cremonte y Botto 2016) mostraron en una muestra restringida de pastas toda la geodiversidad existente en el lugar y la intención de su uso y manipulación, así como la presencia de vasijas foráneas por encontrarse en una vía de circulación entre la quebrada de Humahuaca y la puna jujeña. Mientras que en otro extremo, en Tafí (un amplísimo valle extremadamente fértil con patrón de instalación disperso, elevado grado de autosuficiencia económica a nivel familiar y donde los pobladores habrían invertido casi todo su tiempo en las prácticas agrícolas), la manufactura cerámica parece haber tenido, por ejemplo, un interés notoriamente más bajo que la talla lítica (evidenciado en la elaboración de menhires, máscaras, morteros, e incluso en el carácter megalítico en las técnicas constructivas de las viviendas). A lo largo de los siglos, las pastas de Tafí se ordenan en una serie gruesa y una serie fina, 
como ha sido registrado en otros asentamientos contemporáneos del NOA. En estos casos y en el amplísimo rango existente entre ellos, actuaron innumerables variables que, en sus combinaciones, van moldeando identidades materiales. No podemos dejar de lado las tradiciones de origen de gran amplitud geográfica, las variables climáticas que estimulan o no una mayor dedicación en la producción alfarera, la circulación a corta y larga distancia de materias primas y/o conocimientos tecnológicos particulares, (Druc 2001, 2013; Arnold 2003) y el valor social y/o de intercambio de las vasijas, ya sea como contenedores de determinados productos o como agentes de las cosmovisiones e intereses sociales.

Volviendo entonces a las comparaciones realizadas, en primer lugar y en referencia al ámbito más cercano a Las Garza, como es el sector de Cabra Corral en el mismo valle de Lerma, pudimos registrar que la pasta de Cabra Corral 4.Lav.1, con casi 10\% de tiesto molido y porcentajes similares de sedimentitas y metamorfitas (6.62\% de limolita cuarzosa y $7.33 \%$ de pizarra), coincide con la modalidad de manufactura de las cerámicas locales de Las Garzas, que incluye, además, fuertes similitudes en las estructuras de los fondos de pasta. Pero no así el resto de las pastas de Cabra Corral; si bien varias de ellas presentan inclusiones no plásticas de naturaleza similar, los fondos de pastas indican el empleo de arcillas diferentes y/o de diferentes tratamientos de las mismas.

En segundo lugar, la pasta Las Garzas 5 con casi 15\% de litoclastos graníticos curiosamente no se asemeja a las pastas San Francisco con granito (Pereyra y Cremonte 2017), como habíamos esperado. Sino que, por la cocción oxidante completa, la estructura del fondo de pasta, la ausencia de tiesto molido, granulometría de las inclusiones no plásticas y por los litoclastos límpidos que presenta, muestra estrechas similitudes con las pastas gruesas del tipo IRC (Castańo rojizo alisado) de Tafí. Por el momento y exclusivamente sobre la base de afinidad petrográfica y de otros comportamientos de manufactura conexos, podemos plantear como hipótesis de trabajo que esta vasija de Las Garzas sea resultado de una conexión directa o indirecta entre los pobladores de ambas áreas, es decir que seguimos sosteniendo su manufactura no local. Por otro lado, no podemos dejar de considerar que pastas semejantes muestran la serie gruesa de la alfarería producida por poblaciones contemporáneas de la falda del Aconquija (Pereyra 2012) pudiendo también existir conexiones con ellos.

En tercer lugar, observamos similitudes entre la pasta 11 de Las Garzas y la del fragmento de vaso Vaquerías de La Ciénega (valle de Tafí). Se trata de pastas finas, compactas, oxidantes con inclusiones de pizarras tabulares, escasas sedimentitas y tiesto, presencia de cavidades grandes y estructura de fondo de pasta fluidal. Si bien Las Garzas 11 presenta inclusiones de granulometría más gruesa, las características texturales de ambas pastas son análogas. En La Ciénega, este fragmento Vaquerías fue encontrado en una tumba huaqueada y es sin duda de manufactura no local, similar a pastas Vaquerías de la Quebrada del Toro (Cremonte 1997), sin que por ello podamos aventurar este posible origen para la vasija de Las Garzas.

Por último, la pasta Las Garzas 18 (Vaquerías) comparte similitudes con las pastas San Francisco bicolor PV09 (ocupación por debajo del Pucara de Volcán, quebrada de Humahuaca) y San Francisco bicolor del sitio Santa María (SM sup 90) del valle del río San Francisco, ambos lugares en la provincia de Jujuy. Todas ellas son pastas finas, de color naranja con agregado de tiesto molido. Como ya fuera dicho en otras oportunidades, estas similitudes nos retrotraen al planteo de Dougherty (1975) en cuanto a que el origen del Vaquerías debe buscarse en el bicolor de San Francisco. Evidentemente, las pastas finas del San Francisco y algunas del Vaquerías reflejan un parentesco que aún no ha podido ser cabalmente interpretado.

En síntesis, en Las Garzas son comunes las pastas gruesas que reflejan componentes locales de la Formación Puncoviscana, con agregado de tiesto molido en un porcentaje considerable y ausencia de la serie fina y gruesa asociadas a recipientes de servicio y de preparación/cocción de alimentos respectivamente, como ocurre en otros ámbitos del NOA. Respecto del tiesto molido, su presencia en más del $50 \%$ de las pastas analizadas se considera como un indicador que vincula esta elección tecnológica de Las Garzas con San Francisco y Vaquerías. Por otro lado, los fragmentos asignables a 
Vaquerías, procedentes del sito Las Garzas como de Cabra Corral en el mismo valle, son frecuentes y puede plantearse la probable manufactura local de algunos, considerando la afinidad existente con la geología local e inmediatamente próxima a los sitios. Otros recipientes, tales como los rojos pulidos gruesos y los vasos cilíndricos pulidos presentan pastas que no se corresponden con las locales, indicando contactos con poblaciones occidentales. La comparación de las pastas de Las Garzas con conjuntos San Francisco, Tafí y Vaquerías fundamentalmente, permitió determinar algunos nexos en las técnicas de manufactura.

\section{REFERENCIAS}

Adams, A.E., W.S, Mackenzie y C. Guilford

1984 Atlas of sedimentary rocks under the microscope, Logmann. Harlow, Essex, 104 Great Britain.

Ambrosetti, J.B.

1906 Exploraciones arqueológicas en la Pampa Grande, en: Publicaciones de la Sección Antropológica de la Revista de la Universidad de Buenos Aires, tomo V, Buenos Aires.

Arnold, D.E.

2003 Ecology and ceramic production in an Andean community, Cambridge University Press, Cambridge.

Baldini, M., E. Baff; M. Salaberry y M. Torres

2003 Candelaria: una aproximación desde un conjunto de sitios localizados entre los cerros de Las Pirguas y El Alto del Rodeo (dto. Guachipas, Salta, Argentina), en: G. Ortiz y B. Ventura (eds.), La mitad verde del mundo andino. Investigaciones arqueológicas en la vertiente oriental de los Andes y las tierras bajas de Bolivia y Argentina, 131- 151, Universidad Nacional de Jujuy, San Salvador de Jujuy.

Balfet, H., M. Fauvet- Berthelot y S. Monzon

1983 Pour la normalisation de la description des poteries. París: Editions du Centre National de la Recherche Scientifique.

Bugliani, M. F.

2009 Métodos de ordenación y técnicas de agrupamiento aplicadas a los recipientes cerámicos: las vasijas de los cementerios de la falda occidental de Aconquija. Arqueometría Latinoamericana. Segundo Congreso Argentino y Primero Latinoamericano., O. Palacios, C. Vázquez, T. Palacios y E. Cabanillas (eds.). Comisión Nacional de Energía Atómica. Buenos Aires, 6 al 8 de junio de 2007. Centro Atómico Constituyentes- (CNEA). Argentina.

Cremonte. M.B.

1997 Investigaciones arqueológicas en la Quebrada de la Ciénega (Dpto. Tafí, Tucumán). Tesis doctoral. Facultad de Ciencias Naturales y Museo. Universidad Nacional de La Plata, ms.

Cremonte. M.B e I.L, Botto

2016 Characterization of pottery fabrics from Cueva de Cristobal (Puna of Jujuy), Vessels Explores: Apply Archaeometry to South American Ceramics and their Production, BAR Publishing, 5-13, Oxford.

Cremonte, M.B. y M.F. Bugliani

2006- Pasta, forma e iconografía. Estrategias para el estudio de la cerámica arqueológica, Xama 19(23), $2009 \quad 239-262$.

Cremonte, M.B., N. Flegenheimer., L.J.M. De Santis

1987 El yacimiento Las Garzas (valle de Lerma, Salta). Boletín del Colegio de Graduados en Antropología (16), 21-28, Argentina.

Childs, S.T.

1991 Style, Technology and Iron Smelting in Bantu Speaking Africa, Journal of Anthropological Archaeology (10), 332-359. https://doi.org/10.1016/0278-4165(91)90006-J 
Dietler, M. y I. Herbich

1998 Habitus, techniques and style: an integrated approach to the social understanding of material culture and boundaries, en: M.T. Stark (ed.), The archaeology of social boundaries, 232-263, Smithsonian Institution Press, Washington, D.C.

\section{Dougherty, B.}

1975 Breve reseña sobre la arqueología del Río San Francisco, primera seriación arqueológica, en: Actas y Trabajos del Primer Congreso de Arqueología Argentina, 363- 382, Museo Histórico Provincial "Dr. Julio Marc", Rosario.

Druc, I.

2001 ¿Shashal o no shashal? esa es la cuestión. Etnoarqueología cerámica en la zona de Huari, Ancash, Bulletin de L'Institut Francais D'etudes Andines 30(1), 157-173. https://doi.org/10.4000/bifea.7349

2013 What is local? Looking at ceramic production in the Peruvian highlands and beyond, Journal of Anthropological Research, 69(4), 485-514. https://doi.org/10.3998/jar.0521004.0069.404

Escobar, J. M.

1996 El período alfarero temprano en el valle de Lerma: El caso del sitio Silisque- Tilián 2 (Departamento Chicoana, Provincia de Salta), Revista del Museo de Historia Natural de San Rafael (23), 33-49, San Rafael.

Feely, A.

2012 El concepto de estilo tecnológico cerámico y su aplicación en un caso de estudio: los grupos formativos del oeste de Tinogasta (Catamarca), Arqueología 18, 49-75.

\section{Garay de Fumagalli, M. y M. B. Cremonte}

2002 Ocupaciones agropastoriles tempranas a sur de la Quebrada de Humahuaca (Jujuy, Argentina), Chungará, Revista de Antropología Chilena 34 (1), 35-52. https://doi.org/10.4067/S0717-73562002000100003

González, A. R.

1963 Las tradiciones alfareras del Período Temprano del N.O. argentino y sus relaciones con las áreas aledañas, Congreso Internacional de Arqueología de San Pedro de Atacama, Anales de la Universidad de Chile 2, Antofagasta.

González, A. R. y V. Núñez Regueiro

1960 Preliminary report on archaeological research in Tafí del Valle, NW Argentina, Actas del XXXIV Congreso Internacional de Americanistas, 485- 496, Viena.

Heredia, O. R.

1970 Investigaciones arqueológicas en el sector meridional de la subárea de las Selvas Occidentales, tesis de doctorado, Facultad de Filosofía y Letras, Universidad Nacional de Córdoba, Córdoba.

Ingold, T.

2000 The perception of the environment. Essays in livelihood, dwelling and skill, Routledge, Londres.

Irurzun, A., N. Larcher, G. Gonzáles y C. G. Gorgonza

2011 Rock-magnetic studies on paleolake sediments from Tajamar Formation, Latinmag letters 1, D25, 1-6.

Larcher, N.E.

2006 Estudio hidrogeológico del sector norte de la ciudad de Salta en relación a la ubicación de un nuevo pozo de abastecimiento de agua en la Universidad Nacional de Salta, tesis profesional, Universidad Nacional de Salta, 101, Salta.

Lazzari, M., L. Pereyra Domingorena., M. C. Scattolin., L. Cecil., M. D. Glascock y R. J. Speakman

2009 Ancient social landscapes of northwestern Argentina: Preliminary results of an integrated approach to obsidian and ceramic provenance, Journal of Archaeological Science 36, 1955-1964. https://doi. org/10.1016/j.jas.2009.05.005

Lemonnier, $P$.

1989 Bark Capes, arrowheads and Concorde: on social representations of technology, en: I. Hodder (ed.), The meanings of things. Material culture and symbolic expression, 156-171, One World Archaeology 6, Unwin Hynan, Londres. 
Ortiz, G

2013 Nuevos avances en relación con las prácticas económicas de los grupos pedemontanos de la cuenca del San Francisco (noroeste de Argentina, 800 a.C.-500 d.C.) a través de marcadores biomoleculares y microrrestos vegetales, Revista Española de Antropología Americana 43(2), 369-384. https://doi.org/10.5209/ rev_REAA.2013.v43.n2.44015

\section{Pereyra, L.}

2012 Manufacturas alfareras al sur de los valles calchaquíes entre el siglo primero al quinto d.C., Relaciones, 387-412.

Pereyra Domingorena L. y M. B Cremonte

2017 The Same Way of Doing Pottery. San Francisco Ceramic Fabrics from Tumbaya (Quebrada de Humahuaca) and San Pedro (San Francisco River Basin), en: A. Scaro, C. Otero y M. Cremonte (eds.), Pre-Inca and Inca Pottery, 1-27, The Latin American Studies Book Series. Springer, Cham. https://doi. org/10.1007/978-3-319-50574-9_1

Ryden, $S$.

1934 Note préliminaire sur lárchéologie de la región de La Candelaria (prov. De Salta, Rep. Argentine), Actas y Trabajos del XXV Congreso Internacional de Americanistas, tomo 2, 149- 164, Buenos Aires.

Scattolin, M. C. y J.M. Gero

1999 Consideraciones sobre fechados radiocarb6nicos de Yutopián (Catamarca, Argentina), Actas del XII Congreso Nacional de Arqueología Argentina, tomo 1 (1l), 352-357. La Plata.

Turner, J.C.M.

1972 Cordillera Oriental, Leanza, en: A.F (ed.), Geología regional argentina, Academica Nacional de Ciencias, 117-142, Córdoba.

Recibido: 31/01/2019

Aceptado: 31/07/2019 